\title{
Os Impactos Provenientes da Adoção de XBRL nos Fatores de Qualidade de Software: uma análise descritiva.
}

\section{The impacts of XBRL adoption on software quality factors: a descriptive analysis}

\author{
Paulo Caetano da Silva1, Marcelo Gomes de Cerqueira2 \\ 1Departamento de Pós-Graduação em Ciência da Computação \\ Universidade Salvador (UNIFACS) \\ Salvador, Bahia - Brasil \\ 2 Departamento de Pós-Graduação em Ciência da Computação \\ Universidade Salvador (UNIFACS) \\ Salvador, Bahia - Brasil \\ paulo.caetano@unifacs.br, marcelo.cerqueira@icloud.com
}

\begin{abstract}
XBRL (eXtensible Business Reporting Language) is a technology currently adopted by various government institutions and companies around the world. Many papers related to its use and benefits for the financial and accounting areas are found in the literature. However, little is known of its benefits for Software Engineering. XBRL can impact on financial software development processes as well as software quality factors. Therefore, there is a need to identify the impacts that arise from the adoption of XBRL in financial software development processes and software quality factors. This paper aims to identify the impacts of adopting XBRL on software quality factors. Identifying these impacts may help software developers to understand the advantages of using XBRL and to increase the adoption of XBRL in other companies which may contribute to improving the quality of the software developed and to better implementation of software quality frameworks within these institutions. In addition, this work will contribute to the identification of the adoption factors of the XBRL language.
\end{abstract}

Keywords. XBRL, Software Quality Factors, Software Engineering, Impacts of XBRL

Resumo. XBRL (eXtensible Business Reporting Language) é uma tecnologia adotada atualmente por várias instituições governamentais e empresas ao redor do mundo. Muitos trabalhos relacionados ao seu uso e beneficios para as áreas financeira e contábil são encontrados na literatura. Entretanto, pouco se conhece dos seus beneficios para a Engenharia de Software. XBRL pode causar impactos nos processos de desenvolvimento de software financeiro, assim como nos fatores de qualidade de software. Logo, há necessidade de se identificar os impactos provenientes da adoção de XBRL nos processos de desenvolvimento de software financeiro e nos fatores de qualidade de software. Este artigo tem como objetivo identificar os impactos provenientes da adoção de XBRL nos fatores de qualidade de software. A identificação desses impactos poderá

Cite as: $\quad$ da Silva, P. C. \& Cerqueira, M. G. (2020). The impacts of XBRL adoption on software quality factors: a descriptive analysis (Os Impactos Provenientes da Adoção de XBRL nos Fatores de Qualidade de Software: uma análise descritiva). iSys: Revista Brasileira de Sistemas de Informação (Brazilian Journal of Information Systems), 13(1), 33-59. 
ajudar as empresas desenvolvedoras de software a conhecer melhor as vantagens relacionadas ao uso de XBRL, a aumentar a adoção de XBRL em outras empresas, o que, consequentemente, poderá contribuir com a melhoria da qualidade dos softwares desenvolvidos, facilitando a implementação de frameworks de qualidade dentro dessas instituições. Além disso, esse trabalho poderá contribuir com a identificação dos fatores de adoção da linguagem $X B R L$.

Palavras-Chave. XBRL, Fatores de Qualidade de Software, Engenharia de Software, Impactos de XBRL.

\section{Introdução}

Muitas pesquisas, relacionada ao uso de XBRL (eXtensible Business Reporting Language), referem-se à investigação dos benefícios relacionados à área financeira e contábil [Blankespoor 2012; Debreceny et al. 2010; Debreceny and Gray 2010; Gomaa et al. 2011; Hwang et al. 2008; Janvrin and Mascha 2010; Liu and Luo and et al. 2014; Liu and Wang and et al. 2014; Shan and Troshani 2014; Srivastava and Kogan 2010; Wang et al. 2014]. Apesar do advento de novas tecnologias, e.g. Big Data, FinTech, Bitcoins, Cyber Security, Machine Learning, Internet of Things e Augmented Reality [Kimber et al. 2015], entre elas a XBRL, e de seu uso de maneira difundida, pouco se conhece sobre os impactos referentes ao uso dessas tecnologias nos fatores de qualidade de software.

Por conseguinte, por falta do conhecimento desses impactos, muitas empresas deixam de ser beneficiadas por novas tecnologias [Debreceny, Roger et al. 2009]. Isto pode afetar negativamente os projetos de desenvolvimento de sistemas financeiros, ou seja, o desenvolvimento de software sem a observância de práticas e metodologias associadas à engenharia de software, as quais poderiam trazer, com a sua internalização, benefícios, e.g. atendimento do prazo de entrega dos projetos de software, aumento da produtividade das equipes de desenvolvimento, melhoria da qualidade do produto de software, redução de custo com o desenvolvimento de sistemas, incremento da reusabilidade, da manutenibilidade, da extensibilidade, da confiabilidade e da testabilidade.

Diante deste cenário, a investigação sobre os impactos provenientes da adoção de XBRL nos fatores de qualidade de software poderá ajudar as empresas desenvolvedoras de aplicações financeiras a melhorar os seus respectivos fatores de qualidade de software: manutenibilidade, flexibilidade, testabilidade, portabilidade, reusabilidade, extensibilidade, interoperabilidade, eficiência, integridade, usabilidade. Além disso, poderá ajudar a melhorar a qualidade dos produtos de software. Ademais, não existem, na literatura, informações a respeito da relação entre XBRL e o desenvolvimento de software [Kloos et al. 2014; Troshani and Lymer 2011].

Este trabalho tem como objetivo investigar os impactos positivos e negativos provenientes da adoção de XBRL nos seguintes fatores de qualidade de software: manutenibilidade, flexibilidade, testabilidade, portabilidade, reusabilidade, extensibilidade, interoperabilidade, eficiência, integridade, usabilidade. Para alcançar esses resultados, foram aplicadas uma pesquisa exploratória e quantitativa através de questionários, aplicados em empresas desenvolvedoras de aplicações financeiras e 
comunidades de usuários da tecnologia XBRL. Os resultados obtidos neste trabalho foram validados através de análises estatísticas.

Este artigo divide-se em cinco seções. Após esta introdução, a qual descreve o contexto e os objetivos deste trabalho, a segunda seção discute a fundamentação teórica necessária para a compreensão do trabalho. Na terceira seção, a metodologia utilizada para o desenvolvimento do trabalho é apresentada. A quarta seção apresenta os resultados obtidos na pesquisa, os detalhes da pesquisa aplicada em empresas que adotaram a linguagem XBRL, as análises estatísticas e as interpretações dos resultados. Enfim, a quinta seção apresenta as considerações finais sobre este trabalho. Além dessas seções, este artigo possui dois apêndices. $O$ apêndice A descreve as fases da pesquisa e os procedimentos utilizados na sua elaboração. O apêndice B apresenta os procedimentos estatísticos utilizados na análise de dados.

\section{Fundamentação Teórica}

Nesta seção são abordados conceitos sobre Fatores de Qualidade e XBRL. Conforme [McCall et al. 1977], fatores de qualidade são um conjunto de características ou condições que contribuem para a qualidade do software. Esses fatores possuem uma característica fundamental: a capacidade de serem mensurados através de métodos específicos. Essas mensurações permitem avaliar o nível de qualidade de um determinado fator. Para isso, são necessários índices referenciais contra os quais os fatores medidos sejam comparados. Com isso, é possível avaliar o nível de qualidade de um determinado fator e, em decorrência disso, a qualidade do software. Segundo McCall, fatores de qualidade proveem de meios formais e consistentes para avaliar e inspecionar produtos de software que são desenvolvidos através de processos. Fatores de qualidade podem ser encontrados em todas as fases de desenvolvimento de software. Podemos encontrá-los nas fases de levantamento de requisitos, design, planejamento, desenvolvimento, testes, implantação e manutenção.

De acordo com [McCall et al. 1977; Wang and Wang 2003], os principais fatores de qualidade são: manutenibilidade, flexibilidade, testabilidade, portabilidade, reusabilidade, extensibilidade, interoperabilidade, confiabilidade, eficiência, integridade e usabilidade [Carroll et al. 2015]. Todos fatores serão utilizados nesta pesquisa.

- Manutenibilidade refere-se à capacidade de localizar e eliminar erros dentro de um programa de computador.

- Flexibilidade está relacionada ao esforço requerido para modificar um programa.

- Testabilidade é o empenho demandado para testar um programa, assegurando que este atenda às funções solicitadas.

- Portabilidade é o esforço demandado para transferir um programa de um computador para outro. Essa transferência também está relacionada ao ambiente operacional do software.

- Reusabilidade é capacidade de um programa ser reutilizado por outros diferentes daqueles para o qual foi projetado. 
- Extensibilidade é a capacidade que um software possui para ser alterado através do acréscimo de novos componentes.

- Interoperabilidade é a capacidade de interagir com outros sistemas.

- Confiabilidade é a capacidade de um sistema executar as funções préestabelecidas com precisão.

- Eficiência é a quantidade de recursos computacionais e códigos requeridos por um programa para executar a sua respectiva função.

- Integridade é a capacidade de acesso ao software e aos dados desse software por pessoas não autorizadas.

- Usabilidade é o esforço requerido para aprender, operar e interpretar um software e seus respectivos resultados.

Segundo [Debreceny, Roger et al. 2009], XBRL é uma tecnologia para comunicação eletrônica de dados financeiros e de negócios que revolucionou a área de relatórios financeiros ao redor do mundo. XBRL oferece benefícios na preparação, na análise e na comunicação de relatórios financeiros. Contudo, existem muitas outras possibilidades de aplicação para a XBRL, e.g. no uso em Big Data. Antes da existência de XBRL, as informações financeiras eram divulgadas através de planilhas eletrônicas, documentos Adobe Acrobat (PDF), blocos de texto e páginas Web. Assim, nos processos de divulgação e recebimento de informações, existiam muitos problemas relacionados à conversão de formatos de dados, devido às restrições impostas pelas empresas recebedoras. Eventualmente, essas informações precisavam ser convertidas para bancos de dados internos, antes de serem submetidas às análises, e, posteriormente, serem reutilizadas em outras aplicações. Nesse processo de conversão de dados, aconteciam muitos erros que, na maioria das vezes, acarretavam em retrabalhos ou na divulgação de informações inconsistentes. XBRL possibilita vários tipos de benefícios a esse cenário e atua na padronização dessas informações, evitando os processos de reformatação de dados e reenvio. Com isso, XBRL evita retrabalhos, diminui custos e reduz o tempo gasto na execução dos processos de conversão.

As principais estruturas tecnológicas da linguagem XBRL são: as especificações, as taxonomias e os documentos de instância. As especificações regulamentam a sintaxe e a semântica para a criação de taxonomias e instâncias. Elas definem os elementos e atributos XBRL, os quais podem ser usados para representarem as informações usadas no processo de intercâmbio entre as empresas divulgadoras e analisadoras. Essas especificações utilizam XML Schema, documentos XML e Xlink [Feng et al. 2002; Silva 2003].

As taxonomias são responsáveis pela representação sintática e semântica dos conceitos financeiros e contábeis, definindo seus termos e relacionamentos. Os documentos de instância são responsáveis por fornecer os fatos ou informações em um contexto específico. Os documentos de instância são vinculados às taxonomias através de Linkbases e de documentos XML Schema. Os documentos XML Schema definem os elementos que representam os conceitos financeiros, i.e. o seu vocabulário. Os Linkbases possibilitam estabelecer o relacionamento entre conceitos definidos em taxonomias, i.e. nos documentos XML Schema [Debreceny, R et al. 2009]. Essas relações são definidas através de atributos Xlink [Feng et al. 2002; Silva 2003]. Os Linkbases usam as 
tecnologias XLink e XPointer [Feng et al. 2002; Silva 2003]. A tecnologia XPointer usa a tecnologia XPath para navegar pelos documentos (W3C, 2014).

Há cinco tipos de Linkbases definidos na especificação XBRL 2.1: calculation, definition, label, reference e presentation. Segundo (Silva et al., 2013), o Linkbase calculation indica como o valor de um conceito é calculado, sendo totalizado a partir da agregação de um conjunto de valores de outros conceitos, cada um com a sua parcela e uma soma. O definition habilita diversos tipos de relacionamentos de especialização de conceitos agrupados. O label é responsável pela documentação dos conceitos e pela definição dos rótulos que serão utilizados nas apresentações dos dados que representam fatos relacionados aos conceitos. Ele permite a globalização dos conceitos com o uso de diversos idiomas. O reference é responsável pelo estabelecimento de relacionamentos entre conceitos e normas. Por fim, o Linkbase presentation é responsável pela distribuição e organização dos conteúdos dentro de uma taxonomia e representa como os conceitos devem ser organizados para fins de apresentação. Essa organização segue a hierarquia do domínio, normalmente utilizam as ligações pai-filho.

\section{Metodologia}

As técnicas de pesquisa adotadas neste trabalho são do tipo quantitativa e exploratória. A pesquisa exploratória foi utilizada para investigar, na literatura, o estágio em que se encontra o tema principal deste trabalho, i.e., o estado da arte. Essa revisão da literatura, denominada Coming Impacts of XBRL Adoption in Financial Software Development Processes and Software Quality Factors: A Systematic Mapping, pode ser encontrada em [Silva and Cerqueira 2016]. A pesquisa quantitativa realiza-se através do uso de questionários ou formulários [Mendon 2013]. Os questionários foram enviados para profissionais da área de engenharia de software e de negócios, i.e., área financeira e de contabilidade, que utilizam ou utilizaram a tecnologia XBRL.

O tipo de estudo utilizado foi o quantitativo, o qual é baseado na definição de hipóteses, na definição de questões de pesquisas e nas análises estatísticas para validação dos estudos. As questões de pesquisa foram definidas após a revisão sistemática na literatura [Silva and Cerqueira 2016]. Não foram utilizados estudos qualitativos, em razão deles permitirem diferentes pontos de vista dos participantes da pesquisa e a descoberta de novos aspectos. Além disso, estudos qualitativos não permitem a formulação de hipóteses antecipadamente e o teste de teorias formuladas [Denzin and Lincoln 2006]. Essas teorias são induzidas durante os estudos [Heitmann and Ohling 2005]. No Apêndice A, a metodologia adotada é descrita em detalhes.

\subsection{Definição das Hipóteses}

A questão de pesquisa foi definida após a revisão da literatura [Silva and Cerqueira 2016] e com base nas recomendações sugeridas por [Creswell 2012; Kitchenham and Charters 2007; Recker 2013] A questão de pesquisa está apresentada na Tabela 1.

A questão de pesquisa acima poderá auxiliar na identificação dos impactos que ocorrem na adoção da linguagem XBRL no desenvolvimento de aplicações financeiras e contábeis. Além disso, essa questão ajudará a identificar se esses impactos favorecem os fatores de qualidade de software e as equipes de desenvolvimento de software. 
Tabela 1 - Questão de pesquisa.

\begin{tabular}{l|l|l}
\hline & Descrição da Questão de Pesquisa & Objetivo da Questão de Pesquisa \\
\hline QP1 & $\begin{array}{l}\text { Na percepção de desenvolvedores e e } \\
\text { usuários de XBRL, quais são os impactos } \\
\text { provenientes da adoção de XBRL nos } \\
\text { fatores de qualidade de software? }\end{array}$ & $\begin{array}{l}\text { Identificar os impactos, positivos e negativos, } \\
\text { provenientes da adoção de XBRL nos fatores de } \\
\text { qualidade de software. }\end{array}$ \\
\hline
\end{tabular}

\subsection{Definição das Hipóteses}

Para se alcançar os objetivos propostos, foi necessária a definição de hipóteses. As hipóteses adotadas são as seguintes:

- H01: É possível, com a adoção de XBRL, produzir impactos positivos ou negativos nos fatores de qualidade de software;

- H02: É possível, com a adoção de XBRL, produzir benefícios para as equipes de desenvolvimento de software financeiro;

- H00 (Nula): Com adoção de XBRL, não existem impactos nos fatores de qualidade de software, como também não existem benefícios para as equipes de desenvolvimento de software financeiro com o uso de XBRL.

Essas hipóteses poderão auxiliar na solução do problema de pesquisa porque a partir delas será possível identificar e comprovar a existência de impactos nos fatores de qualidade de software com a adoção de XBRL. Além disso, essas hipóteses poderão comprovar a existência de benefícios para as equipes de desenvolvimento de software financeiro com a adoção da linguagem XBRL no desenvolvimento de aplicações financeiras e contábeis.

As hipóteses serão testadas através de procedimentos de estatística descritiva, fundamentados em [Field 2013; Hair et al. 2010]. Nessa etapa, também serão utilizados procedimentos metodológicos baseados em [Creswell 2012; Kitchenham and Pfleeger 2002; Recker 2013] Serão aplicados na análise de dados as seguintes funções estatísticas: moda, média, desvio padrão, variação, regressão e coeficiente alfa. Os apêndices A e B apresentam detalhes adicionais sobre a coleta de dados

\section{Resultados}

Os questionários aplicados neste trabalho foram enviados, aproximadamente, para 55 instituições que desenvolvem softwares baseados em XBRL ou fazem uso de XBRL para o intercâmbio de dados financeiros. Foram feitos contatos prévios através de telefone e e-mails com os responsáveis pela divulgação dos questionários nas instituições. A abordagem de contatar o responsável pela divulgação da pesquisa, na instituição, dificultou a identificação do número de respondentes que receberam os questionários e não os responderam. Um total de 30 pessoas responderam todas as perguntas dos questionários. Esse resultado representa um índice estimado de resposta de 54\%. Como parâmetro de comparação, nas pesquisas relacionadas à Engenharia de Software, aplicadas por [Kautz and Larsen 1996] e [Stålhane et al. 1997], foram obtidas uma taxa de resposta de $13.3 \%$ para a pesquisa de Kautz e $8.4 \%$ para a pesquisa de Stålhane. As 
pesquisas de Kautz e Stålhane utilizaram procedimentos semelhantes aos procedimentos aplicados neste trabalho.

\subsection{Perfil dos participantes}

Os questionários foram distribuídos para desenvolvedores de software e usuários da tecnologia XBRL de diversos países no período entre outubro de 2015 e abril de 2016. Um total de 9 países participaram desta pesquisa.

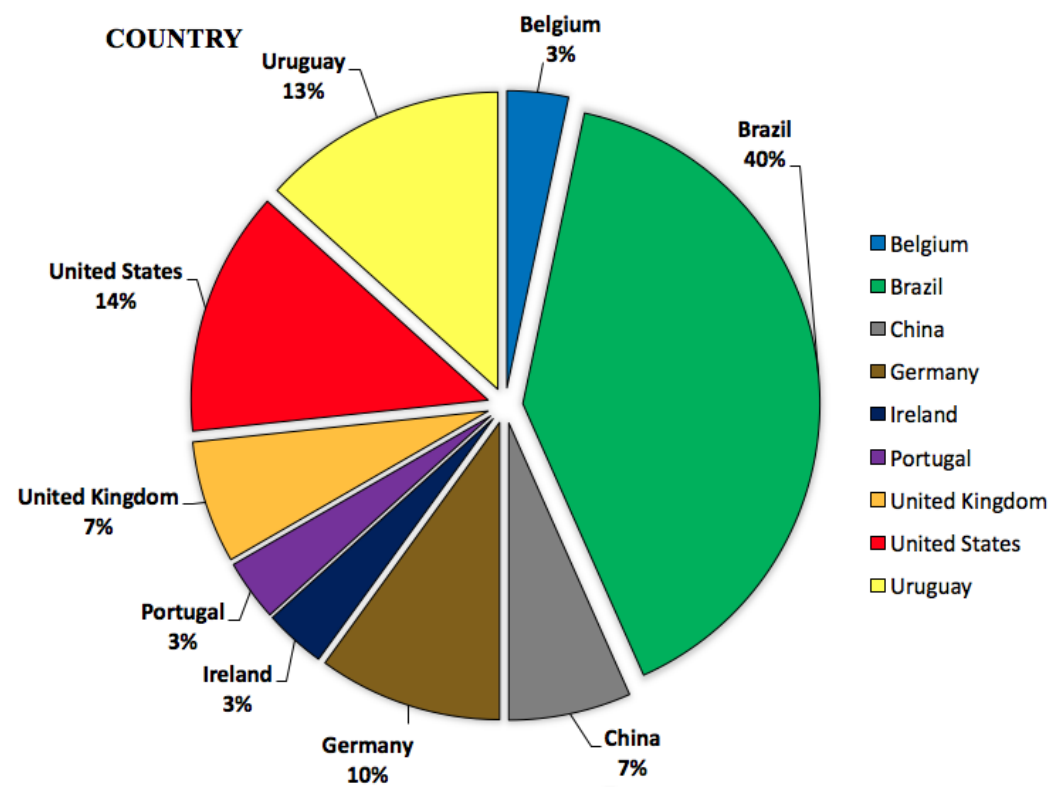

Figura 1 - Países que participaram da pesquisa. Fonte: 0 autor.

Os países participantes da pesquisa foram: Brasil (40\%), Estados Unidos (14\%), Uruguai (13\%), Alemanha (10\%), Reino Unido (7\%), China (7\%), Bélgica (3\%), Irlanda (3\%), Portugal (3\%).

A maioria dos participantes na pesquisa é proveniente de Brasília-DF, Montevideo, Frankfurt, Beijing, Summerville e São Paulo-SP. Nas demais cidades, houve a participação de um respondente por cidade (Dublin, London, Fortaleza-CE, Lisboa, Mülheim an der Ruhr, Nevele, Oxford, Recife-PE e Salvador-BA.)

Dos profissionais que responderam sobre suas respectivas atuações, atualmente, $68,96 \%$ estão trabalhando na área de Tecnologia da Informação. Os outros respondentes (31,03\% do total) estão atuando na área de Negócios.

A análise dos resultados deste trabalho não foi generalizada, isto é, considerando os dois grupos (Tecnologia da Informação e Negócios), pois a generalização dos resultados não permitiria caracterizar o comportamento diferente entre cada grupo de profissionais. Além disso, a estratificação dos resultados em duas camadas reduz o erro amostral, melhorando a precisão dos resultados. Essa separação foi utilizada nos gráficos para uma melhor análise dos resultados.

Sobre o tempo de experiência com o uso de XBRL, 46.67\% dos participantes responderam que usam XBRL entre 1 e 5 anos. $40.00 \%$ informaram que trabalham com 
XBRL há mais de 5 anos. 6.67\% responderam que usam XBRL a menos de um ano. Em um total de $100 \%$ dos participantes, $6.67 \%$ não responderam a essa pergunta.

Acerca dos números de empregados das empresas que fizeram parte da pesquisa através dos respondentes, $63.33 \%$ possuem mais de 500 empregados, $13.33 \%$ possuem entre 100 e 499 empregados, $13.33 \%$ possuem entre 20 e 99 empregados, $6.66 \%$ não responderam a essa pergunta e $3.33 \%$ possuem menos que 20 empregados.

\subsection{Impactos nos Fatores de Qualidade de Software.}

Nesta seção serão apresentados os resultados referentes ao questionário relacionado aos impactos nos fatores de qualidade de software.

Questão 1: Após a adoção de XBRL, como você classifica o seu impacto na reusabilidade de software.

Resultados: Os profissionais da área de TI $(58,62 \%$ do total) consideraram que a adoção de XBRL produz impactos positivos ou muito positivos no fator reusabilidade. Aqueles da área de negócios $(24,13 \%$ do total) responderam conforme os da área de TI. A razão dessa escolha pode ser associada à capacidade da linguagem XBRL ser reutilizada por outros softwares por causa da compatibilidade de tipo de dados, facilidade de utilização de informações provenientes de estruturas XBRL e pela capacidade de reuso das taxonomias. Além disso, a integração de XBRL com Web Services permite novas formas de reusabilidade [Debreceny et al. 2007]. A Figura 2 mostra as frequências obtida nessa questão através dos questionários referente aos Fatores de Qualidade de Software.

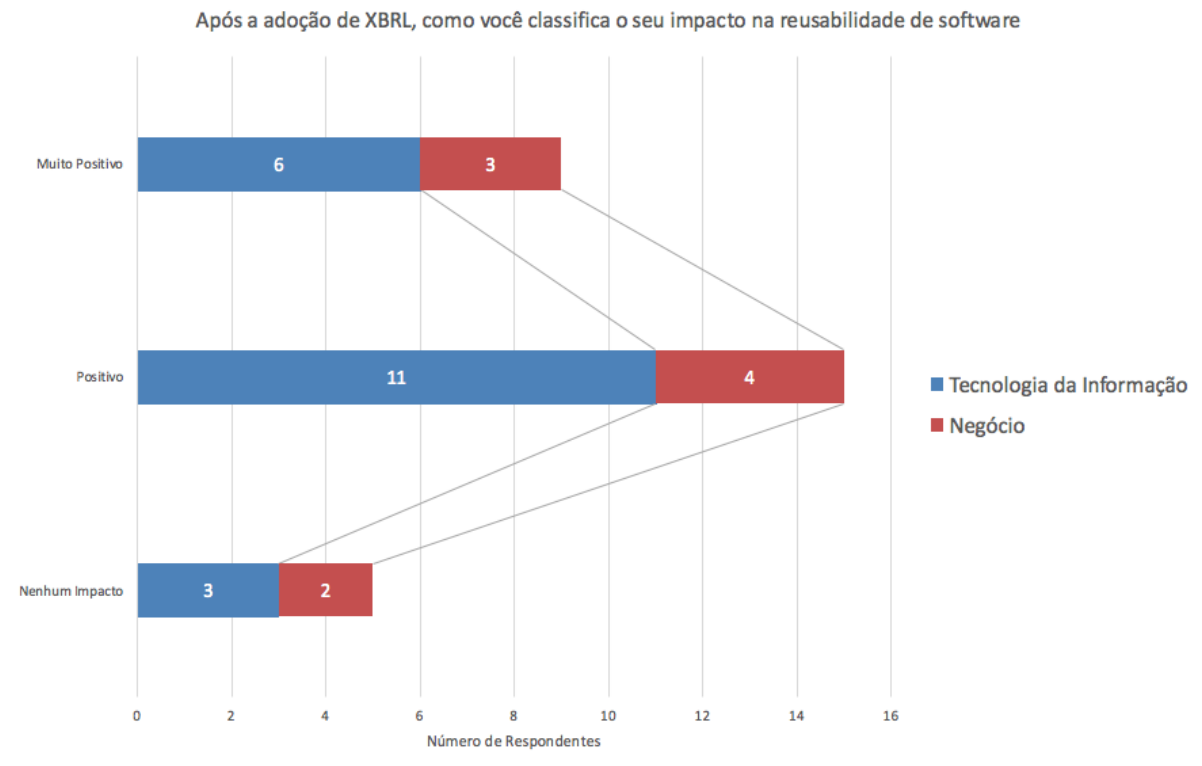

Figura 2 - Frequências da questão 1: Após a adoção de XBRL, como você classifica o seu impacto na reusabilidade de software.

Questão 2: Após a adoção de XBRL, como você classifica o seu impacto na usabilidade de software.

Resultado: Os profissionais da área de TI (37,93\% do total) consideraram que a adoção de XBRL não produz impactos na usabilidade de software. Já os profissionais da área de negócio $(13,79 \%$ do total) responderam que XBRL causa impactos positivos na 
usabilidade de software. A resposta dada pelos profissionais da área de negócios pode estar associada à usabilidade obtida nos relatórios financeiros [Liu and Wang and et al. 2014]. Na Figura 3 são apresentadas as frequências obtidas nesta questão.

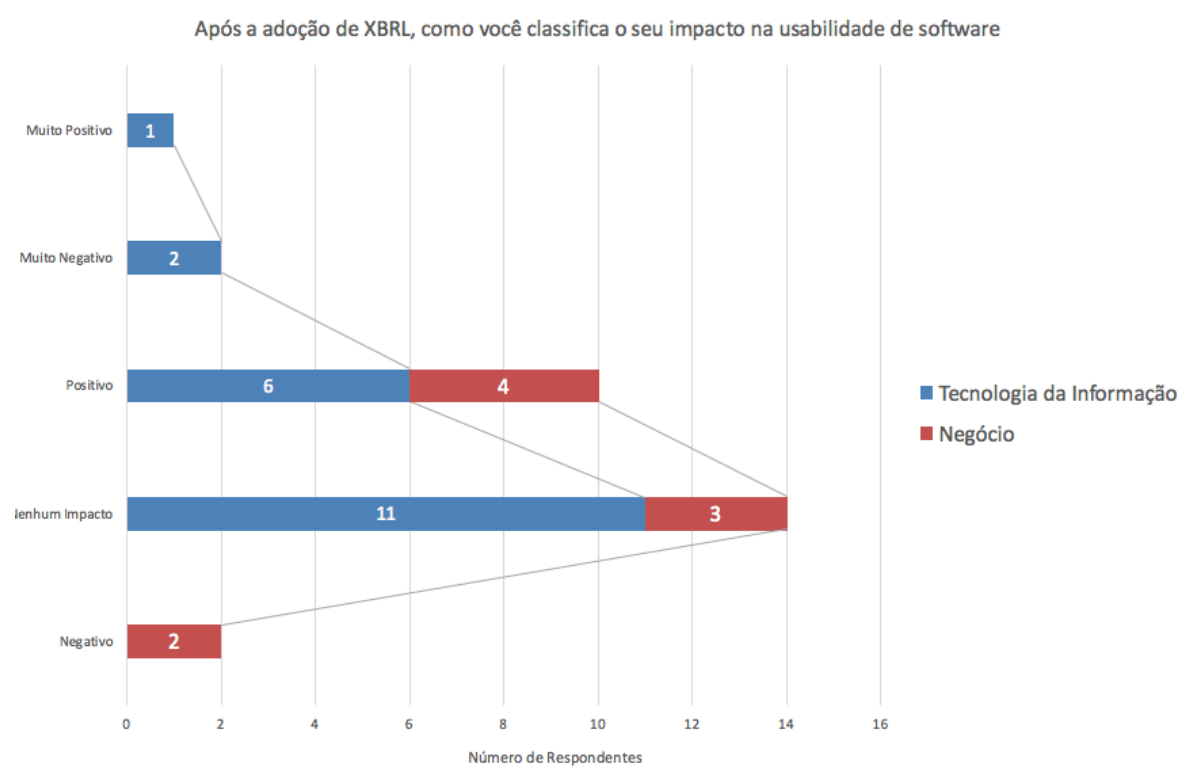

Figura 3 - Frequências da questão 2: Após a adoção de XBRL, como você classifica o seu impacto na usabilidade de software.

Questão 3: Após a adoção de XBRL, como você classifica o seu impacto no fator segurança.

Resultados: Os profissionais da área de TI (48,27\% do total) responderam que XBRL não causa impactos no fator segurança. A resposta dos profissionais da área de negócios foi de acordo com os da área de TI. A justificativa dessa resposta pode ser relacionada à falta de evidências empíricas sobre a contribuição de XBRL para o fator segurança. Na revisão sistemática da literatura não foram encontradas informações relacionadas ao fator segurança. Na Figura 4 são mostradas as frequências desses resultados.

Questão 4: Após a adoção de XBRL, como você classifica o seu impacto no fator confiabilidade.

Resultados: Os participantes da área TI $(41,37 \%$ do total) responderam que XBRL causa impactos positivos ou muito positivos no fator confiabilidade. Os participantes da área de negócios $(17,24 \%$ do total) responderam conforme os participantes da área de TI. A razão dessa resposta pode ser justificada pelos benefícios que XBRL produz a padronização de informações, a melhoria da qualidade de dados, a redução de erros nos processos de validação de taxonomias e fórmulas, a redução de informações redundantes e a facilidade de integração com outros sistemas ou pacotes de software. Todas essas razões podem acarretar no aumento da confiabilidade das soluções que adotam XBRL. Na Figura 5 são demostradas as frequências dessa pergunta. 


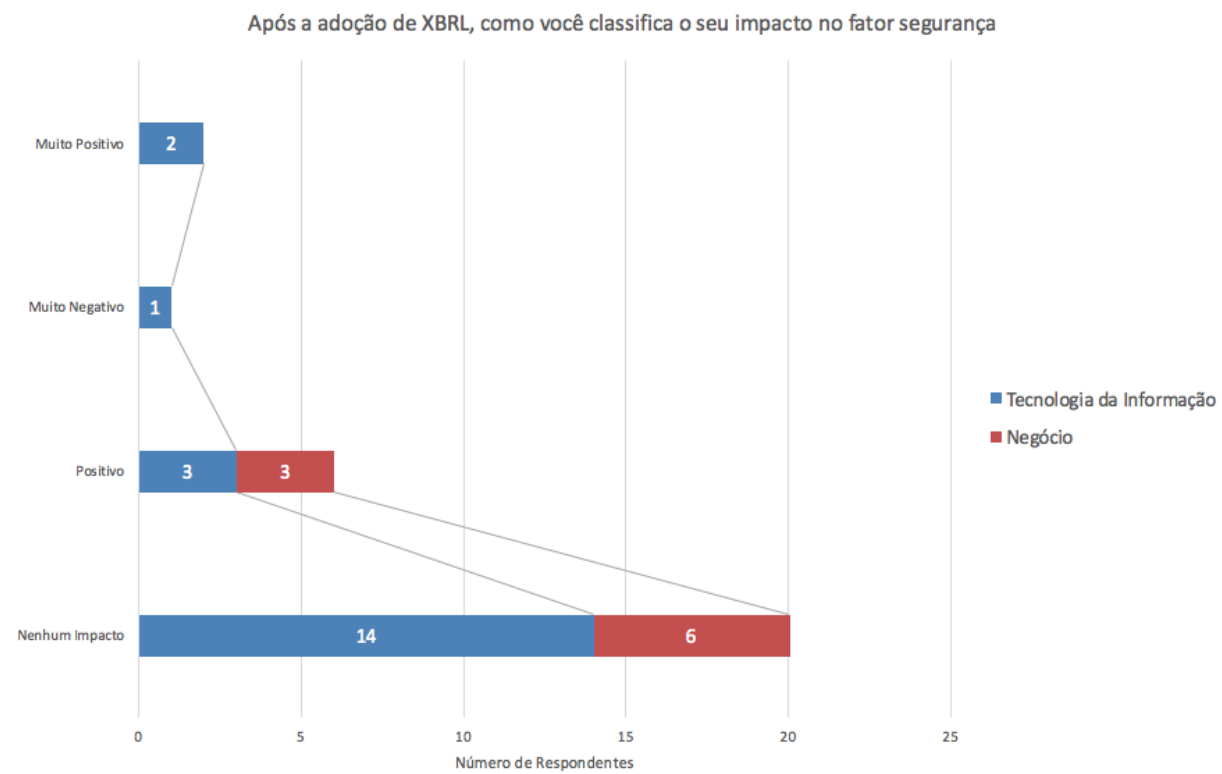

Figura 4 - Frequências da questão 3: Após a adoção de XBRL, como você classifica o seu impacto no fator segurança.

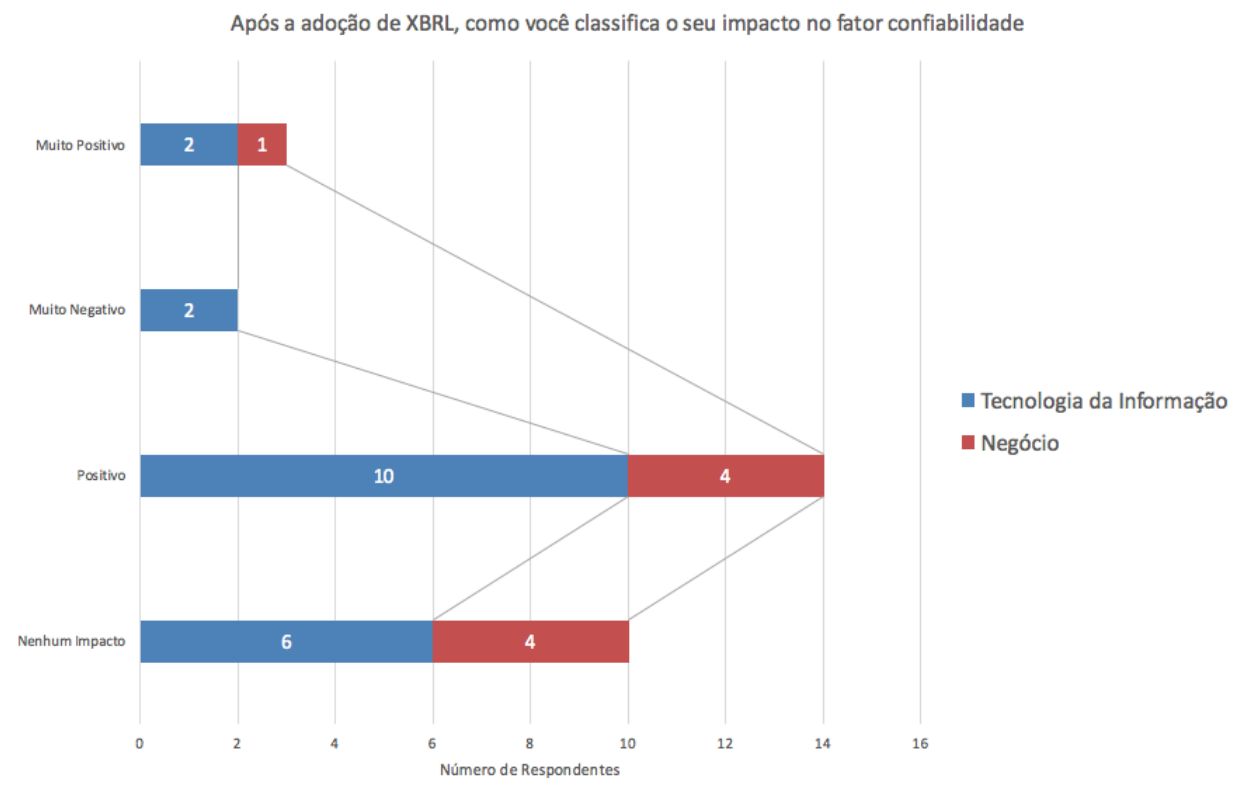

Figura 5 - Frequências da questão 4: Após a adoção de XBRL, como você classifica o seu impacto no fator confiabilidade.

Questão 5: Após a adoção de XBRL, como você classifica o seu impacto no fator extensibilidade.

Resultado: Os participantes da área de TI $(62.06 \%$ do total) consideraram que XBRL causa impacto positivos ou muito positivos no fator extensibilidade. A resposta dos participantes da área de TI coincide com a escolha da área de negócios $(14,13 \%$ do total). A justificativa dessa resposta pode estar relacionada à capacidade que XBRL possui para definir novas estruturas através da criação de novos elementos [Silva 2003]. 
Além desse resultado, foram encontradas evidências na revisão sistemática sobre a relação positiva entre XBRL e o fator extensibilidade [Davidson et al. 2006; Pinsker and Li 2008]. A Figura 6 mostra as frequências de respostas obtidas nessa questão.

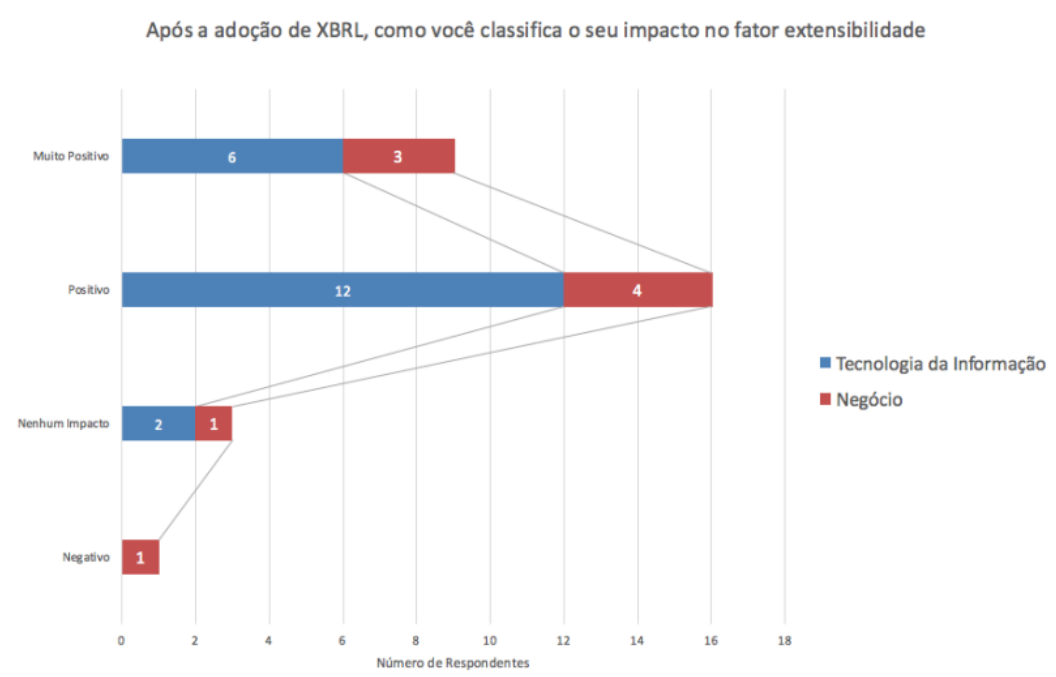

\section{Figura 6 - Frequências da questão 5: Após a adoção de XBRL, como você} classifica o seu impacto no fator extensibilidade.

Questão 6: Após a adoção de XBRL, como você classifica o seu impacto no fator manutenibilidade

Resultado: Os profissionais da área de TI $(41,37 \%$ do total) consideraram que XBRL causa impactos positivos ou muito positivos no fator manutenibilidade. Os profissionais da área de negócios $(20,68 \%$ do total) responderam conforme os profissionais da área de TI. A razão dessa escolha pode estar associada às propriedades semânticas de XBRL, que facilitam à identificação de erros e à redução do esforço de programação e nos testes de software. Na Figura 7 são apresentadas as frequências desse resultado.

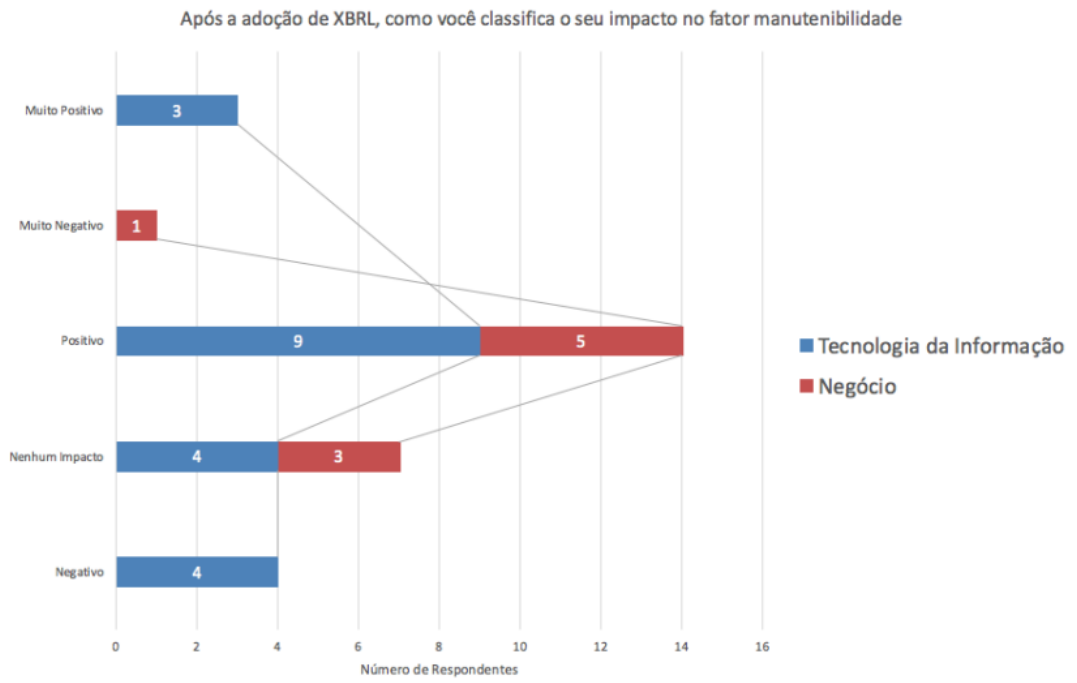

Figura 7 - Frequências da questão 6: Após a adoção de XBRL, como você classifica o seu impacto no fator manutenibilidade. 
Questão 7: Após a adoção de XBRL, como você classifica o seu impacto no fator flexibilidade.

Resultado: Os participantes da área de TI (34,48\% do total) responderam que XBRL não produz impactos no fator flexibilidade. Os participantes da área de negócios $(17,24 \%$ do total) consideraram que XBRL causa impactos positivos no fator flexibilidade. A justificativa para a escolha dos participantes da área de negócios pode ser associada à facilidade de realizar modificações em uma taxonomia e instância XBRL. Entretanto, para fornecer essa facilidade, as taxonomias XBRL precisam ser bem projetadas [Hoffman and Watson 2009]. Na Figura 8 são mostradas as frequências obtidas com essa questão.

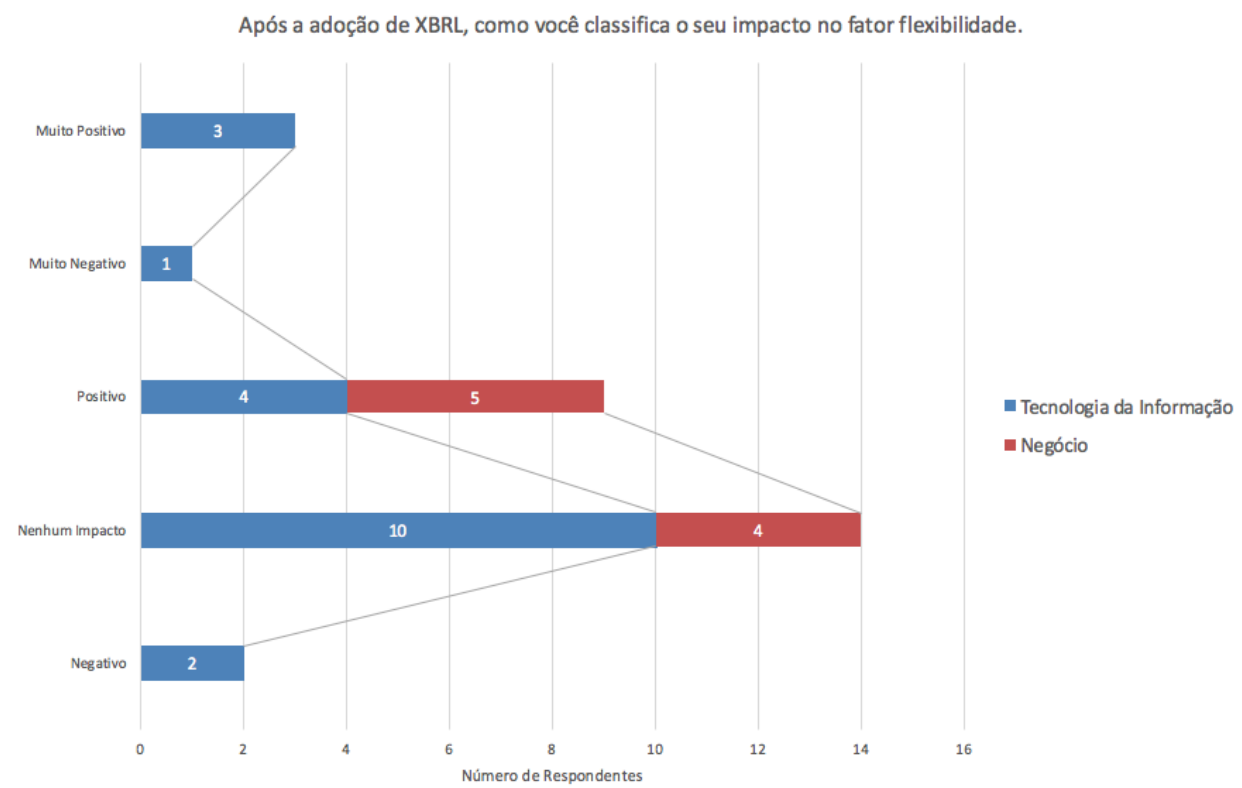

Figura 8 - Frequências da questão 7: Após a adoção de XBRL, como você classifica o seu impacto no fator flexibilidade.

Questão 8: Após a adoção de XBRL, como você classifica o seu impacto no fator testabilidade.

Resultado: Os profissionais da área de TI $(55,17 \%$ do total) consideraram que XBRL causa impactos positivos ou muito positivos no fator testabilidade. Os profissionais da área de negócios $(24,13 \%$ do total) responderam conforme os profissionais da área de TI. Esse resultado confirma a resposta da questão 08 (Após a adoção de XBRL, como você classifica o impacto na execução de testes de software), a qual obteve resultado também positivo. A justificativa dessa escolha pode ser associada às justificativas da questão 08 (propriedades semânticas, processos de validação, redução de códigos de programação através da associação de metadados e a forma que os dados são estruturados em XBRL). Na Figura 9 são apresentadas as frequências dos resultados dessa questão. 


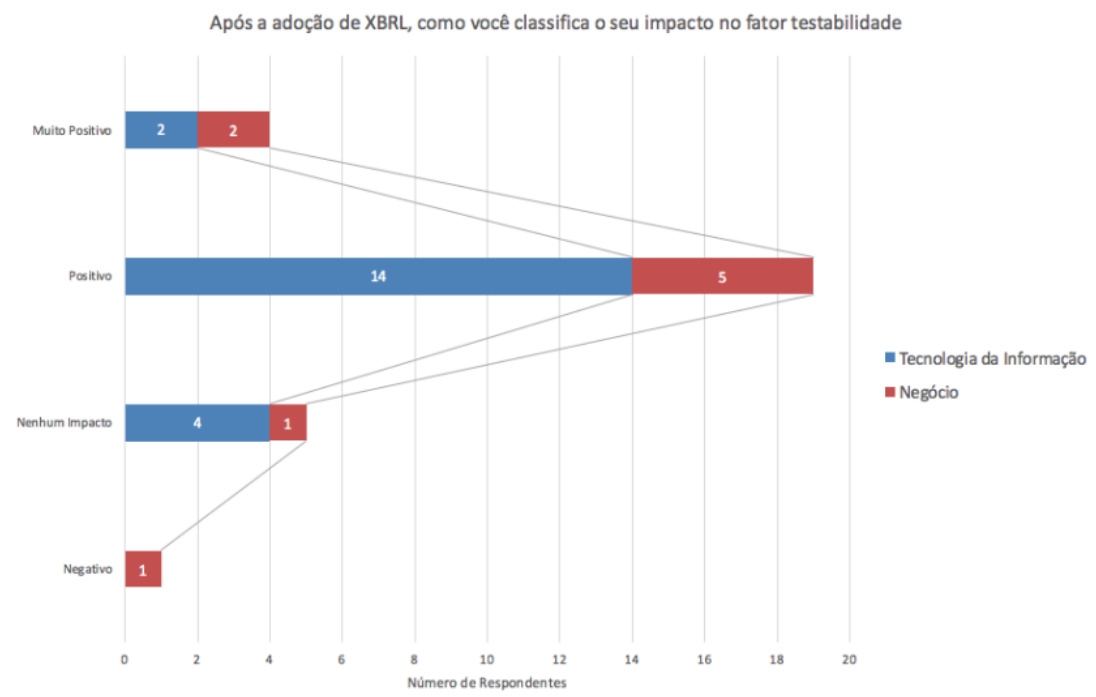

Figura 9 - Frequências da questão 8 - Após a adoção de XBRL, como você classifica o seu impacto no fator testabilidade.

Questão 9: Após a adoção de XBRL, como você classifica o seu impacto no fator portabilidade.

Resultados: Os participantes da área de TI (48,27\% do total) consideraram que XBRL causa impactos positivos no fator portabilidade. Os participantes da área de negócios $(24,13 \%$ do total) responderam conforme os participantes da área de TI. A razão desse resultado pode ser associada à capacidade de XBRL ser usada em diferentes plataformas operacionais, devido à herança da linguagem XML. Essa herança facilita a transferência de informações entre diferentes plataformas operacionais. Além disso, XBRL pode ser integrado com Web Services, oferecendo outras opções de portabilidade para sistemas hospedados em Mainframes [Debreceny et al. 2007]. Na Figura 10 são apresentadas as frequências desse resultado.

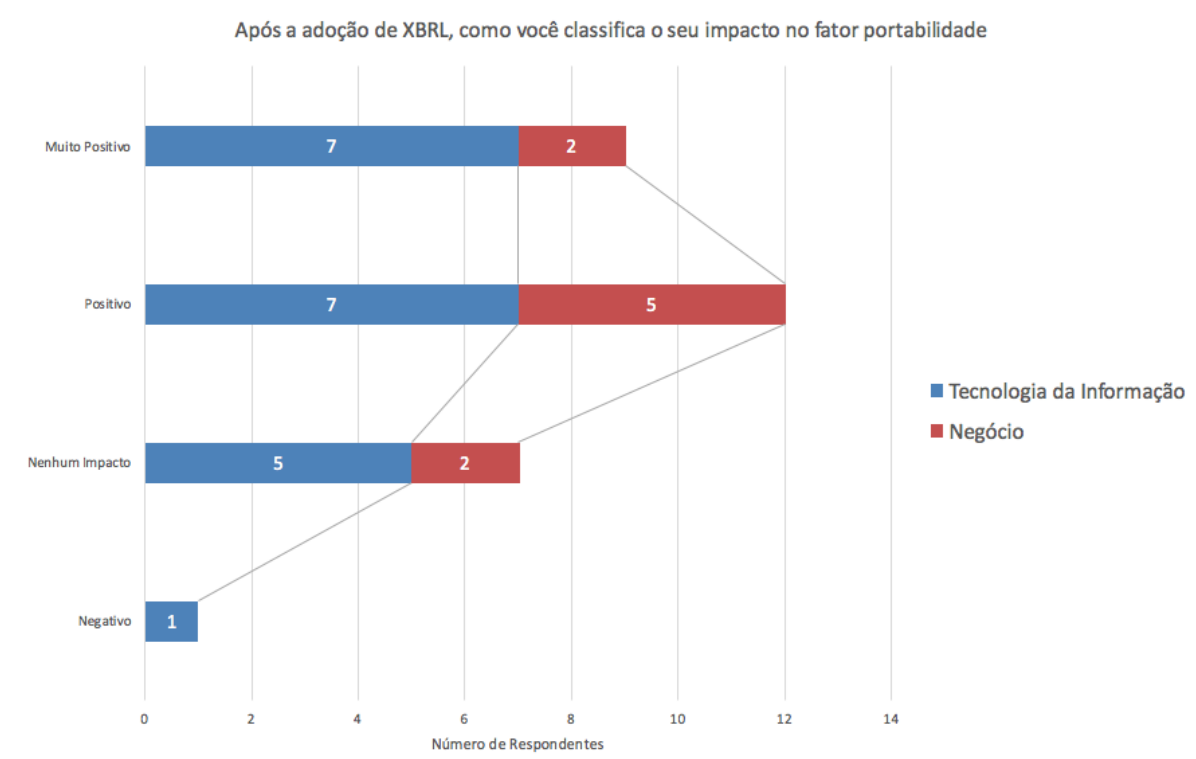

Figura 10 - Frequências da questão 9: Após a adoção de XBRL, como você classifica o seu impacto no fator portabilidade. 
Questão 10: Após a adoção de XBRL, como você classifica o seu impacto no fator Interoperabilidade.

Resultado: Os profissionais da área de TI $(55,17 \%$ do total) consideraram que XBRL causa impactos positivos ou muito positivos no fator interoperabilidade. Os profissionais da área de negócios $(27,58 \%$ do total) responderam conforme os profissionais da área de TI. Esse resultado confirma a resposta da questão 11 (Após a adoção de XBRL, como você classifica o impacto na integração de XBRL com outras ferramentas ou pacotes de software). A razão dessa escolha pode ser relacionada à facilidade de integração de XBRL com outros softwares, por causa da sua capacidade semântica, que facilita a troca de informações entre banco de dados de diferentes plataformas operacionais. Além disso, XBRL trabalha com especificações. As empresas que desejam adotar XBRL precisam aderir a essas especificações, as quais facilitam o intercâmbio de informações entre sistemas diferentes. A Figura 11 exibe as frequências dessa questão.

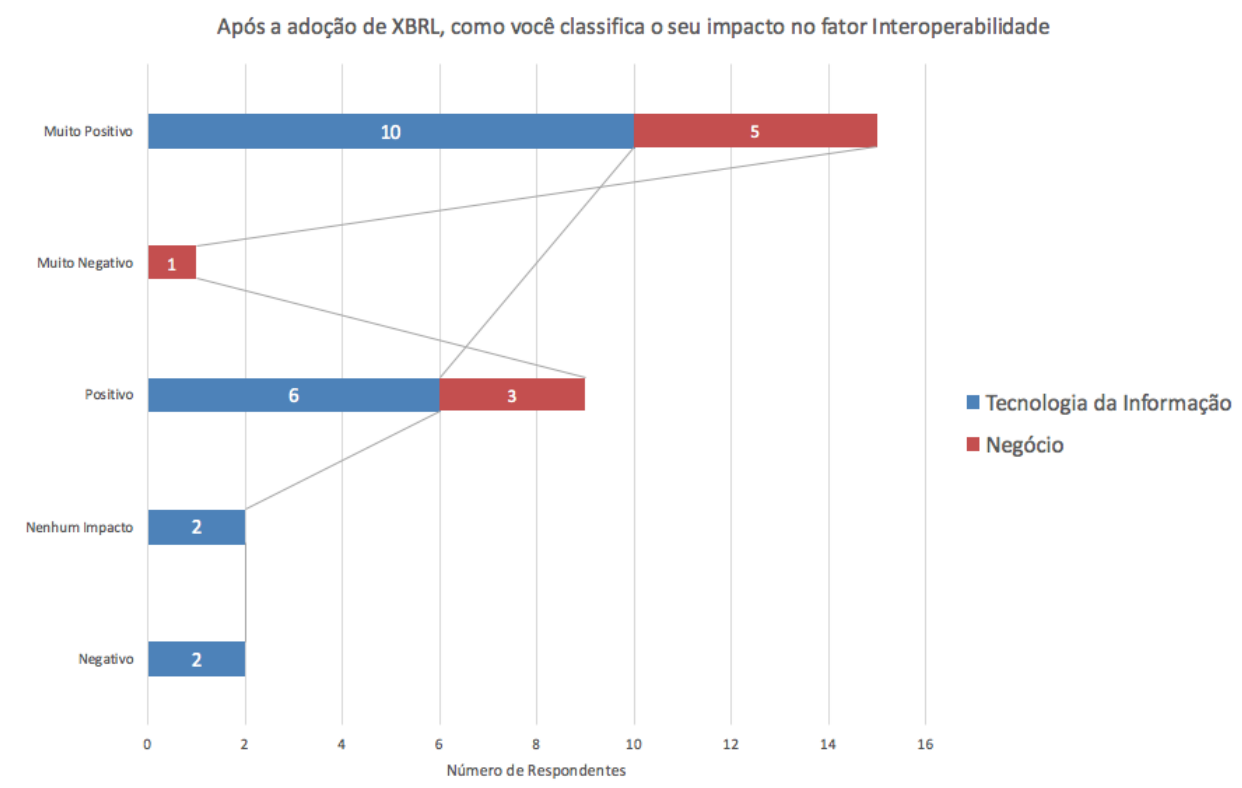

Figura 11 - Frequências da questão 10: Após a adoção de XBRL, como você classifica o seu impacto no fator Interoperabilidade.

Questão 11: Após a adoção de XBRL, como você classifica o seu impacto na Integridade de dados.

Resultados: Os resultados obtidos pelos participantes da área de TI (55,17\% do total) indicaram que a adoção XBRL causa impactos positivos ou muito positivos na integridade de dados. Os participantes da área de negócios $(20,68 \%$ do total) consideraram a mesma indicação da área de TI. A razão dessa escolha pode ser associada à forma que XBRL padroniza as informações, que favorece a objetividade, a capacidade de análise (esforço necessário para encontrar erros) e a integridade das informações. XBRL não permite controle de acesso aos seus dados ou proteção de dados submetidos através da Internet. Além disso, XBRL não tem como garantir que um documento recebido é o mesmo enviado, ou seja, não se garante que o documento não sofreu manipulações no processo de transferência de dados, portanto, é necessário se 
implementar medidas de segurança para o intercâmbio da informação representada em XBRL [Escobar-Rodríguez and Gago-Rodríguez 2012]. Na Figura 12 são demostradas as frequências dessa questão.

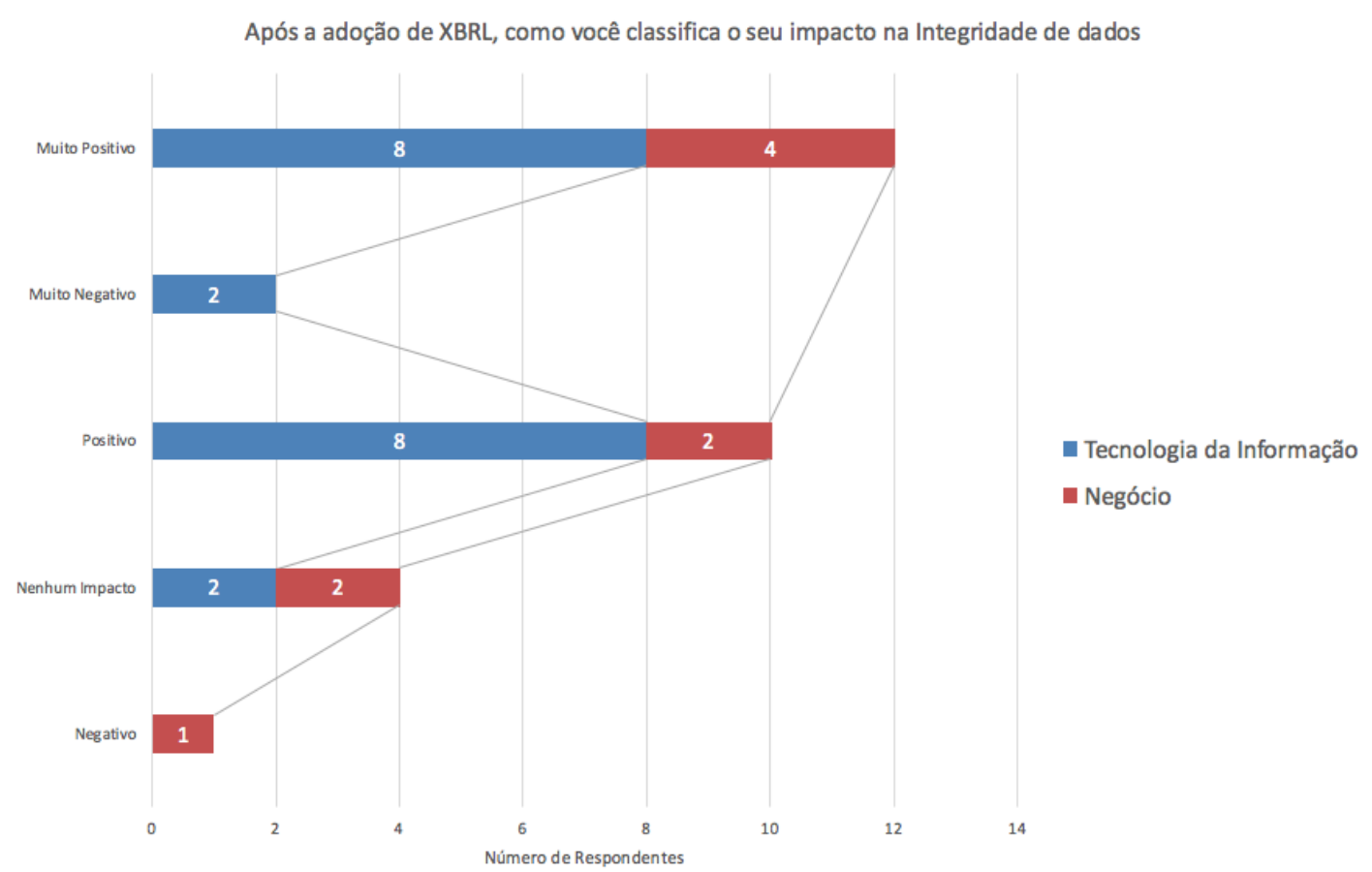

Figura 12 - Frequência da questão 11: Após a adoção de XBRL, como você classifica o seu impacto na Integridade de dados.

\subsection{Definição das Hipóteses}

$\mathrm{Na}$ análise de consistência de dados, foi aplicado o Coeficiente Alfa (Cronbach's Alpha). O resultado desse teste deve oscilar entre o número 0 e 1. Segundo [Dyba 2000], resultados acima de 0.78 demostra um alto nível de consistência, entre 0.55 e 0.77 são considerados de consistência moderada e abaixo de 0.55 são considerados de baixa consistência. Seguindo a mesma metodologia aplicada em Dyba, as análises de consistência dos resultados foram executadas no SPSS (Software e Linguagem para Análises Preditivas da IBM). Dyba considerou o SPSS como um software confiável para a execução desse tipo de análise.

"...An internal consistency analysis was performed for each of the six key facilitating factors of SPI success using the SPSS reliability program (SPSS, 1999a, 1999b)" [Dyba 2000]

$\mathrm{Na}$ Tabela 2, são apresentados os resultados obtidos nessa análise, utilizando o Coeficiente Alfa. 
Tabela 2 - Coeficiente Alfa do questionário sobre os Impactos nos Fatores de Qualidade de Software. Fonte: SPSS.

\begin{tabular}{|c|c|c|c|c|c|}
\hline \multicolumn{6}{|l|}{ Item-Total Statistics } \\
\hline & $\begin{array}{l}\text { Scale Mean if } \\
\text { Item Deleted }\end{array}$ & $\begin{array}{l}\text { Scale Variance } \\
\text { if Item Deleted }\end{array}$ & $\begin{array}{l}\text { Corrected } \\
\text { Item-Total } \\
\text { Correlation } \\
\end{array}$ & $\begin{array}{l}\text { Squared } \\
\text { Multiple } \\
\text { Correlation }\end{array}$ & $\begin{array}{l}\text { Cronbach's } \\
\text { Alpha }\end{array}$ \\
\hline $\begin{array}{l}\text { Após a adoção de } \\
\text { XBRL, como você } \\
\text { classifica o seu } \\
\text { impacto na } \\
\text { reusabilidade de } \\
\text { software }\end{array}$ & 37.14 & 39.266 & .366 & .519 & .876 \\
\hline $\begin{array}{l}\text { Após a adoção de } \\
\text { XBRL, como você } \\
\text { classifica o seu } \\
\text { impacto na } \\
\text { usabilidade de } \\
\text { software }\end{array}$ & 38.07 & 34.924 & .674 & .754 & .857 \\
\hline $\begin{array}{l}\text { Após a adoção de } \\
\text { XBRL, como você } \\
\text { classifica o seu } \\
\text { impacto no fator } \\
\text { segurança }\end{array}$ & 38.00 & 39.571 & .295 & .595 & .880 \\
\hline $\begin{array}{l}\text { Após a adoção de } \\
\text { XBRL, como você } \\
\text { classifica o seu } \\
\text { impacto no fator } \\
\text { confiabilidade }\end{array}$ & 37.72 & 34.635 & .662 & .746 & .858 \\
\hline $\begin{array}{l}\text { Após a adoção de } \\
\text { XBRL, como você } \\
\text { classifica o seu } \\
\text { impacto no fator } \\
\text { extensibilidade }\end{array}$ & 37.14 & 36.195 & .691 & .827 & .858 \\
\hline $\begin{array}{l}\text { Após a adoção de } \\
\text { XBRL, como você } \\
\text { classifica o seu } \\
\text { impacto no fator } \\
\text { manutenibilidade }\end{array}$ & 37.79 & 33.599 & .730 & .739 & .853 \\
\hline $\begin{array}{l}\text { Após a adoção de } \\
\text { XBRL, como você } \\
\text { classifica o seu } \\
\text { impacto no fator } \\
\text { flexibilidade }\end{array}$ & 37.90 & 35.525 & .611 & .686 & .862 \\
\hline $\begin{array}{l}\text { Após a adoção de } \\
\text { XBRL, como você } \\
\text { classifica o seu } \\
\text { impacto no fator } \\
\text { testabilidade }\end{array}$ & 37.38 & 37.887 & .553 & .740 & .867 \\
\hline $\begin{array}{l}\text { Após a adoção de } \\
\text { XBRL, como você } \\
\text { classifica o seu } \\
\text { impacto no fator } \\
\text { portabilidade }\end{array}$ & 37.28 & 36.350 & .575 & .749 & .864 \\
\hline $\begin{array}{l}\text { Após a adoção de } \\
\text { XBRL, como você } \\
\text { classifica o seu } \\
\text { impacto no fator } \\
\text { Interoperabilidade }\end{array}$ & 37.07 & 33.352 & .672 & .672 & .857 \\
\hline $\begin{array}{l}\text { Após a adoção de } \\
\text { XBRL, como você } \\
\text { classifica o seu } \\
\text { impacto na } \\
\text { Integridade de } \\
\text { dados }\end{array}$ & 37.28 & 33.778 & .575 & .601 & .867 \\
\hline
\end{tabular}


Os resultados apresentados nas Tabela 2 revelam-se satisfatórios acerca da consistência e da confiabilidade dos dados obtidos. A média aritmética dos coeficientes individuais (Cronbach's Alpha) foi 0.785 para os impactos referentes aos fatores de qualidade de software. Ambos resultados indicam um nível de consistência ideal. [Almeida et al. 2010] consideram um nível de consistência ideal quando o valor do coeficiente alfa oscila em torno de 0.70 e 0.90 . Valores abaixo de 0.70 significa que os dados apresentam baixa consistência (não confiáveis). Valores acima de 0.90 significa redundância de informação ou duplicidade. [Streiner 2003] considera resultados confiáveis valores entre 0.80 e 0.90 . Um outro argumento acerca da consistência calculada é o coeficiente alfa de todas as variáveis (coeficiente Alfa Geral) referente aos impactos nos processos de desenvolvimento e aos fatores de qualidade de software. $\mathrm{O}$ coeficiente Alfa Geral é calculado no SPSS, através dos resultados obtidos nos coeficientes individuais. A Figura 13 mostra o Coeficiente Alfa Geral, obtido no questionário referente aos impactos nos fatores de qualidade de software.

Tabela 3 - Coeficiente Alfa Geral das 11 variáveis ( $\mathrm{N}$ of Items) referente aos impactos nos fatores de qualidade de software. Fonte: SPSS.

Reliability Statistics

\begin{tabular}{|c|c|c|}
\hline $\begin{array}{c}\text { Cronbach's } \\
\text { Alpha }\end{array}$ & $\begin{array}{c}\text { Cronbach's } \\
\text { Alpha Based on } \\
\text { Standardized } \\
\text { Items }\end{array}$ & $\mathrm{N}$ of Items \\
\hline .875 & .874 & 11 \\
\hline
\end{tabular}

Ambos valores obtidos estão dentro da faixa de 0.80 até 0.90 . Esses resultados revelam um nível satisfatório no cálculo, utilizando o Coeficiente Alfa em todas as variáveis referentes aos Fatores de Qualidade de Software.

\subsection{Interpretação dos Resultados}

As hipóteses de 1 até 2 foram testadas através das técnicas de análise descritiva. Os resultados apresentados nos gráficos (análise descritiva) e na Tabela 2 são evidências empíricas da refutação da hipótese nula (H00). Essa refutação é representada pela proposição abaixo.

$\omega_{1}=$ Existem impactos positivos ou negativos nos fatores de qualidade.

$\omega_{2}=$ Não existe impacto nos processos de desenvolvimento e nos fatores de qualidade.

Se $\omega_{1}>\omega_{2}$, então $H 1=$ validada

Se $\omega_{1}=\omega_{2}$, então $H O O=$ validada

Os resultados obtidos na pesquisa foram:

$\left(\omega_{1}>\omega_{2}\right)-$ Logo, os estudos suportam as hipóteses alternativas.

\section{Refutação da hipótese nula (H00).}


No que concerne aos dados da pesquisa, os testes de Cronbach's (Coeficiente Alfa) apresentaram altos valores (acima de 0.70) nas 11 variáveis. Os resultados do Coeficiente Alfa estão apresentados nas Tabelas 2. Esses resultados indicam consistência e confiabilidade nos dados obtidos nesta pesquisa. Os valores desses resultados foram calculados utilizado o SPSS versão 22. A justificativa para escolha do SPSS deve-se às evidências empíricas da confiabilidade desse software para a execução desses cálculos [Dyba 2000].

Os resultados provenientes das análises de tendência (Estatística Descritiva) indicam que a adoção de XBRL produz impactos positivos nos seguintes fatores: reusabilidade, confiabilidade, extensibilidade, manutenibilidade, testabilidade, portabilidade, interoperabilidade e na integração de dados. Não houve registro de impactos negativos com o uso de XBRL nos fatores de qualidade de software. Esses resultados respondem à questão de pesquisa $01(\mathrm{QP} 1)$, que trata dos impactos nos fatores de qualidade de software. As resposta das QP1 suporta as hipóteses H01 e H02. A resposta da H02 dá-se através do uso do método indutivo [Marconi and Lakatos 2003] e da seguinte proposição:

Se XBRL produz impactos positivos nos fatores de qualidade de software, então o uso de XBRL produz beneficios para as equipes de desenvolvimento de software financeiro.

\subsection{Ameaças à Validade}

Algumas ameaças à validade do trabalho foram detectadas. Entretanto, tais questão poderão ser dirimidas em trabalhos futuros.

A primeira questão que se coloca diz respeito ao envolvimento dos respondentes com a tecnologia, i.e., isso induziria a respostas que apenas explicitassem benefícios trazidos pelo seu uso nos fatores da qualidade de software? Todavia, a análise sobre os benefícios ou malefícios não pode ser feita a partir de quem não tem conhecimento da tecnologia. Certamente, essa ameaça pode ser mitigada com uma maior amostra de pesquisa.

A segunda questão refere-se ao viés "social desirability e "automation bias" [Fisher 1993], uma vez que as questões foram apresentadas de forma direta e afirmativa. Entretanto, há a necessidade de se fazer questões de forma objetiva para não se desviar o foco da pesquisa. Porém, para continuidade do trabalho e melhor validação, podem ser adicionadas questões indiretas e sem escalas para mensuração, mitigando o "social desirability e "automation bias".

Outra ameaça refere-se à não utilização de análise inferencial (qui-quadrado) nos resultados obtidos, utilizando uma amostragem estratificada. A utilização da análise inferencial (qui-quadrado) seria prejudicada, devido ao tamanho das amostras (30 respondentes agrupados em duas amostras). A não utilização do qui-quadrado poderá trazer prejuízos na avaliação das hipóteses (interpretação dos resultados).

\section{Conclusão}

Este artigo teve como objetivo a investigação dos impactos provenientes da adoção de XBRL nos fatores de qualidade de software. Além disso, este trabalho buscou investigar os benefícios da adoção de XBRL para as equipes de desenvolvimento de software 
financeiro através de métodos indutivos. Para isso, foi realizada uma pesquisa exploratória, a qual foi aplicada em grupos de pesquisas, empresas de desenvolvimento de aplicações financeiras e comunidades XBRL com o objetivo de obter respostas para as questões de pesquisas definidas neste trabalho.

As análises efetuadas nos resultados suportam as hipóteses $\mathrm{H} 01$ e H02, ou seja, a adoção de XBRL causa impactos positivos e negativos nos fatores de qualidade de software. Além disso, a adoção de XBRL também produz benefícios para as equipes de desenvolvimento de software financeiro. As respostas às questões de pesquisas estão apresentadas abaixo:

\section{- QP1 (Identificar os impactos, positivos e negativos, provenientes da adoção de XBRL nos fatores de qualidade de software):}
$\checkmark$ Impactos positivos: na reusabilidade, na confiabilidade, na extensibilidade, na manutenibilidade, na testabilidade, na portabilidade, na interoperabilidade e na integridade de dados;
$\checkmark$ Impactos negativos: no fator segurança.

A partir deste trabalho pode-se concluir que a adoção de XBRL traz benefícios para as equipes de desenvolvimento de software financeiro, para as empresas de desenvolvimento de soluções financeiras e para o desenvolvimento de soluções integradas com outros sistemas. Além disso, esses resultados poderão contribuir para a identificação dos fatores de adoção da linguagem XBRL, para o entendimento dos benefícios relacionados à adoção de XBRL no desenvolvimento de soluções financeiras e contábeis, para o desenvolvimento frameworks e IDE's que poderão fazer a integração com as especificações XBRL e para a integração de metodologias de engenharia de software nos sistemas de informações financeiros e contábeis. Portanto, a adoção de XBRL também pode facilitar a obtenção de certificações de qualidade de software em razão desses possíveis benefícios proporcionados.

Trabalhos futuros: os impactos negativos identificados neste trabalho poderão ser utilizados como objeto de estudo para o desenvolvimento de soluções. Ademais, poderão ser acrescentados a esse estudo a Análise de Componentes Principais (PCA), principalmente, nos fatores Confiabilidade e Flexibilidade, e, além disso, este estudo poderá ajudar no desenvolvimento de metodologias para implantação de XBRL nos processos de desenvolvimento de software financeiro. Este trabalho será replicado para um desenvolvimento de uma pesquisa sobre os impactos da adoção de XBRL nos processos de desenvolvimento de software financeiro.

\section{Referências}

Almeida, D., Santos, M. A. R. Dos and Costa, A. F. B. (2010). Aplicação do Coeficiente Alfa de Cronbach nos Resultados de um Questionário para Avaliação de Desempenho da Saúde Pública. XXX Encontro Nacional De Engenharia De Produção, p. 1-12.

An Evaluation of the Current State and Future of XBRL and Interactive Data for Investors and Analysts (2012). Whitepaper, Columbia University, Center for Excellence in Accounting \& Security Analysis,

Blankespoor, E. A. (2012). The Impact of Investor Information Processing Costs on Firm Disclosure Choice: Evidence from the XBRL Mandate. 
Boslaugh, S. and Watters, P. (2008). Statistics In a Nutshell: A Desktop Quick Reference. 1th. ed. United States: O'Reilly. v. 1

Carroll, C., Falessi, D., Forney, V., et al. (2015). A Mapping Study of Software Causal Factors for Improving Maintenance. In International Symposium on Empirical Software Engineering and Measurement.

Cleophas, T. J. and Zwinderman, A. H. (2016). SPSS for Starters and 2nd Levelers.

Cortina, J. M. (1993). What is coefficient alpha? An examination of theory and applications. Journal of Applied Psychology, v. 78, n. 1, p. 98-104.

Couper, M. P., Tourangeau, R. and Steiger, D. M. (2001). Social presence in web surveys. Proceedings of the SIGCHI ..., n. 3, p. 412-417.

Creswell, J. W. (2012). Educational research: Planning, conducting, and evaluating quantitative and qualitative research. 4th. ed. Nebraska: Pearson Education. v. 3

Cronbach, L. J. (1951). Coefficient alpha and the internal structure of tests. Psychometrika, v. 16, n. 3, p. 297-334.

Davidson, A., Robinson, A. and Malthus, S. (2006). Knowledge and use of XBRL by Chartered Accountants in New Zealand.

Debreceny, R., Farewell, S., Piechocki, M., Felden, C. and Gräning, A. (2010). Does it add up? Early evidence on the data quality of XBRL filings to the SEC. Journal of Accounting and Public Policy, v. 29, n. 3, p. 296-306.

Debreceny, R., Felden, C. and Ochocki, B. (2009). XBRL Taxonomy Extensions. XBRL for Interactive ...,

Debreceny, R., Felden, C., Ochocki, B., Piechocki, M. and Piechocki, M. (2009). XBRL for Interactive Data.

Debreceny, R., Felden, C. and Piechocki, M. (2007). New dimensions of business reporting and $X B R L$. Wiesbaden: DUV.

Debreceny, R. S. and Gray, G. L. (2010). Data mining journal entries for fraud detection: An exploratory study. International Journal of Accounting Information Systems, v. 11, n. 3, p. 157-181.

Denzin, N. K. and Lincoln, Y. S. (2006). Introduction The Discipline and Practice of Qualitative Research. Handbook of Qualitative Research, p. 1-20.

Dyba, T. (2000). An Instrument for Measuring the Key Factors of Success in Software Process Improvement. Empirical Software Engineering, v. 5, p. 357-390.

Escobar-Rodríguez, T. and Gago-Rodríguez, S. (2012). "We were the first to support a major is innovation". Research into the motivations of spanish pioneers in XBRL. Revista de Contabilidad, v. 15, n. 1, p. 91-108.

Feng, L., Chang, E. and Dillon, T. (2002). A semantic network-based design methodology for XML documents. ACM Transactions on Information Systems, v. 20, n. 4, p. 390421.

Field, A. (2013). Discovering Statistics using IBM SPSS Statistics. Discovering Statistics using IBM SPSS Statistics, p. 297-321. 
Field, A. (2009). Discovering Statistics Using SPSS. THIRD EDIT ed. London: SAGE Publications Ltd.

Fisher, R. J. (1993). Social Desirability Bias and the Validity of Indirect Questioning. Journal of Consumer Research,

Gomaa, M. I., Markelevich, A. and Shaw, L. (jun 2011). Introducing XBRL through a financial statement analysis project. Journal of Accounting Education, v. 29, n. 2-3, p. $153-173$.

Gräning, A., Felden, C. and Piechocki, M. (2011). Status Quo and Potential of XBRL for Business and Information Systems Engineering. Business \& Information Systems Engineering

Günther, H. (2003). Como Elaborar um Questionário. Planejamento de Pesquisa nas Ciências Sociais, n. 1999, p. 1-35.

Hair, J. F., Black, W. C., Babin, B. J. and Anderson, R. E. (2010). Multivariate Data Analysis. Vectors

Heitmann, S. and Ohling, A. (2005). Audit of the Future - An Analysis of the Impact of XBRL on Audit and Assurance.

Hoffman, C. and Watson, L. (2009). XBRL for Dummies.

Hwang, J. S., Leem, C. S. and Moon, H. J. (2008). A study on relationships among accounting transparency, accounting information transparency, and XBRL. Proceedings - 3rd International Conference on Convergence and Hybrid Information Technology, ICCIT 2008, v. 1, n. 0, p. 502-509.

Janvrin, D. and Mascha, M. F. (2010). The Process Of Creating XBRL Instance Documents: A Research Framework. The Review of Business Information Systems, v. 14, n. 2, p. 11-34.

Kautz, K. and Larsen, E. A. (1996). Quality assurance and software process improvement in Norway. In 4th International Conference on the Software Process.

Kimber, A., Angwin, M., Miles-Khan, S. and Backeberg, M. (2015). Emerging Technology Trends. p. 1-7.

Kitchenham, B. and Charters, S. (2007). Guidelines for performing Systematic Literature reviews in Software Engineering Version 2.3. Engineering, v. 45, n. 4ve, p. 1051.

Kitchenham, B. and Pfleeger, S. L. (2002). Principles of survey research part 4: questionnaire evaluation. ACM SIGSOFT Software Engineering Notes, v. 27, n. 3, p. 20.

Kloos, M., Hulstijn, J., Seck, M. and Janssen, M. (2014). XBRL-driven business process improvement: A simulation study in the accounting domain. In Lecture Notes in Computer Science (including subseries Lecture Notes in Artificial Intelligence and Lecture Notes in Bioinformatics).

Liu, C., Luo, X., Sia, C. L., O'Farrell, G. and Teo, H. H. (2014). The impact of XBRL adoption in PR China. Decision Support Systems, v. 59, n. 1, p. 242-249.

Liu, C., Wang, T. and Yao, L. J. (2014). XBRL's impact on analyst forecast behavior: An empirical study. Journal of Accounting and Public Policy, v. 33, n. 1, p. 69-82. 
Marconi, M. and Lakatos, E. (2003). Fundamentos de metodologia científica.

McCall, J. a., Richards, P. K. and Walters, G. F. (1977). Factors in Software Quality. at'l Tech. Information Servicel, v. 1, 2 and 3, n. ADA049055, p. 689-1699.

Mendon, M. (2013). Gismália Marcelino Mendonça Manual de Normalização para Apresentação de Trabalhos Acadêmicos.

Mesquita, M., Salvador, U., Silva, C., et al. (2013). Similarity Evaluation Between Concepts Represented by XBRL. p. 1889-1910.

Petrillo, F., Dal, C., Freitas, S. and Pimenta, M. (2011). Interactive Analysis of Likert Scale Data using a Multichart Visualization Tool. IHC - Simpósio Brasileiro sobre Fatores Humanos em Sistemas Computacionais, p. 358-365.

Pinsker, R. and Li, S. (2008). Costs and benefits of XBRL adoption. Communications of the $A C M$, v. 51, n. 3, p. 47-50.

Recker, J. (2013). Scientific Research in Information Systems. 1th Editio ed. London: Springer.

Robertson, J. (2012). Likert-type scales, statistical methods, and effect sizes. Communications of the ACM, v. 55, n. 5, p. 6.

Shan, Y. and Troshani, I. (2014). Does XBRL benefit financial statement auditing? Journal of Computer Information Systems,

Silva, P. C. (2003). Explorando Linguagens de Marcação para Representaçaõ de Relatórios de Informações Financeiras. v. 1, p. 1-135.

Silva, P. C. and Cerqueira, M. (2016). COMING IMPACTS OF XBRL ADOPTION IN FINANCIAL SOFTWARE DEVELOPMENT PROCESSES AND SOFTWARE QUALITY FACTORS: A SYSTEMATIC MAPPING. [CONTECSI, Ed.]In CONTECSI USP - International Conference on Information Systems and Technology Management. . FEAUSP.

Srivastava, R. P. and Kogan, A. (2010). Assurance on XBRL instance document: A conceptual framework of assertions. International Journal of Accounting Information Systems, v. 11, n. 3, p. 261-273.

Stålhane, T., Borgersen, P. C. and Arnesen, K. (jul 1997). In search of the customer's quality view. Journal of Systems and Software, v. 38, n. 1, p. 85-93.

Streiner, D. L. (2003). Being inconsistent about consistency: when coefficient alpha does and doesn't matter. Journal of personality assessment, v. 80, n. 3, p. 217-22.

Troshani, I. and Lymer, A. (2011). Institutionalizing XBLR in the UK: An Organizing Vision Perspective. ECIS 2011 Proceedings, p. Paper 152.

Vieira, H. C., Castro, A. E. De and Shuch Junior, V. F. (2010). O uso de questionários via e-mail em pesquisas acadêmicas sob a ótica dos respondentes. In: XIII Seminários em Administração - SEMEAD, n. 2006, p. 13.

Wang, H. and Wang, C. (2003). Taxonomy of security considerations and software quality. Communications of the $A C M$, v. 46, n. 6, p. 75-78.

Wang, T., Wen, C. Y. and Seng, J. L. (2014). The association between the mandatory adoption of XBRL and the performance of listed state-owned enterprises and non- 
state-owned enterprises in China. Information and Management, v. 51, n. 3, p. 336346.

\section{Apêndice A - Fases e Procedimentos para a Pesquisa}

O desenvolvimento deste trabalho ocorreu em três etapas. A primeira etapa foi realizada por meio de uma pesquisa de campo, conhecida como survey, que teve como objetivo identificar os impactos provenientes da adoção de XBRL nos processos de desenvolvimento de software financeiro e nos fatores de qualidade de software. A segunda etapa foi a realização de análises estatísticas nos dados obtidos no survey. Enfim, a terceira etapa foi a interpretação dos dados obtidos do survey com o objetivo de responder as questões de pesquisas e validar ou refutar as hipóteses definidas neste artigo.

A primeira etapa constituiu em uma pesquisa realizada através de questionários estruturados que seguiram recomendações de [Creswell 2012], [Marconi and Lakatos 2003] e [Gräning et al. 2011]. Esses questionários foram disponibilizados na Web em comunidades e grupos de estudos que usam ou usaram a tecnologia XBRL com o objetivo de identificar os impactos provenientes da adoção de XBRL nos processos de desenvolvimento de software financeiro e nos fatores de qualidade de software. A distribuição dos formulários da pesquisa foi realizada na Web para grupos de pesquisa e discussão sobre XBRL. Além disso, foram distribuídos formulários para profissionais que já trabalham com a tecnologia XBRL, na tentativa de garantir que as respostas sejam provenientes de pessoas com conhecimento em XBRL. Esses métodos foram baseados nas recomendações de [Couper et al. 2001] e [Vieira et al. 2010]. Os dados provenientes dos formulários foram cadastrados e processados no SPSS (programa para processamento estatístico de pesquisa da IBM) [Field 2009] para inferência das questões de pesquisas.

Com a obtenção dos dados, realizou-se as análises, definidas como a segunda etapa do desenvolvimento deste artigo. Esses dados foram analisados através de procedimentos de estatística descritiva e inferencial, fundamentados em [Field 2013]. Nessa etapa, também foram utilizados procedimentos metodológicos baseados em [Creswell 2012] e [Kitchenham and Pfleeger 2002]. Foram aplicadas nos dados as seguintes funções estatísticas: moda, média, desvio padrão, variação, regressão, coeficiente alfa, coeficiente de independência e correlação bivariada. Os resultados da aplicação dessas funções serão descritos nos parágrafos seguintes. Os gráficos utilizados para a análise dos resultados foram também gerados através do SPSS [Cleophas and Zwinderman 2016].

A quinta etapa foi a interpretação dos dados obtidos na etapa 4 com o objetivo de responder as questões de pesquisa e validar ou refutar as hipóteses definidas neste trabalho.

Segundo [Marconi and Lakatos 2003], a pesquisa tem um papel fundamental na ciência. A mesma é utilizada na obtenção de respostas para questões de pesquisa, validação de hipóteses e na solução de problemas científicos. De acordo com [Günther 2003], para obter resposta às questões de pesquisa e aos problemas científicos, são utilizados os seguintes instrumentos: observação de comportamento, experimento e levantamento de dados (survey). Nesse trabalho utilizamos o survey para dar respostas aos questionamentos científicos. Survey é um método para coletar informações de pessoas acerca de suas opiniões. O mesmo utiliza o questionário como instrumento. 
Questionário é um instrumento de coleta de informações através de perguntas ordenadas [Marconi and Lakatos 2003]. Essas perguntas são respondidas sem a presença do entrevistador. Conforme Lakatos e Marconi existem vantagens e desvantagens associadas ao uso de questionários. As vantagens associadas ao uso de questionários são:

•economia de tempo gasto com locomoção até o local da pesquisa;

-maior abrangência geográfica;

-economia de recursos humanos com trabalho de campo;

• obtenção de respostas mais rápidas e precisas;

-maior liberdade nas respostas, devido ao anonimato;

-inexistência de viés;

•maior disponibilidade de tempo para responder ao questionário em horários mais flexíveis.

As desvantagens relacionadas à aplicação de questionário são:

-possibilidade de perguntas sem respostas;

- impossibilidade de ajudar nas respostas às questões mal compreendidas;

- difículdade de compreensão a qual leva a respostas uniformes;

•distribuição do questionário ao público-alvo.

Entretanto, muitas dessas desvantagens foram mitigadas neste trabalho. $\mathrm{Na}$ possibilidade de perguntas sem respostas, foram adotadas as seguintes ações: foram oferecidos incentivos (uma calculadora HP12C e um Tablet) aos profissionais pesquisados para responder todas as perguntas. A norma de ética 6.14 da American Psychological Association (APA) proíbe apenas incentivos financeiros excessivos ou impróprios. Sobre a impossibilidade de ajudar nas respostas às questões mal compreendidas, foi dado suporte com relação as dúvidas referentes às questões. Esse suporte foi através de e-mails e telefone. Referente à distribuição do questionário ao público-alvo, o questionário foi enviado apenas para profissionais que trabalham com XBRL, participantes de grupos de pesquisa em XBRL e de comunidades de usuários de XBRL. Essas medidas foram tomadas para assegurar a validade e a acurácia dos resultados.

A pesquisa está contextualizada nas áreas de desenvolvimento de aplicações financeira e negócios. A mesma teve a sua distribuição nas principais capitais brasileiras e em territórios internacionais, a pesquisa foi enviada para os seguintes países: Estados Unidos, Chile, Argentina, Uruguai, Espanha, Portugal, Alemanha, Irlanda, Singapura, Rússia, Reino Unido, Suíça e Bélgica.

As premissas adotadas para a elaboração das questões a serem realizadas aos pesquisados foram: coletar informações sobre impactos provenientes da adoção de XBRL nos processos de desenvolvimento de software financeiro e nos fatores de qualidade de software, coletar informações sobre o perfil das pessoas pesquisadas e o perfil das suas respectivas empresas.

De acordo com [Marconi and Lakatos 2003], um questionário deve ser limitado, isto é, o mesmo não pode ser muito longo ou curto, pois há o risco de não oferecer informações suficientes ou causar fadiga e desinteresse. Assim, um questionário deve ter entre 20 a 30 questões e demorar, no máximo, 30 minutos para ser completamente 
respondido. Neste trabalho, o questionário foi composto por 30 questões, sendo que 22 referiam-se às questões de pesquisa, e as 8 restantes aos perfis dos pesquisados e de suas respectivas empresas. Lakatos e Marconi também recomendam que sejam realizados prétestes para avaliar os seguintes elementos: Fidedignidade, Validade e Operatividade. Fidedignidade está relacionada à obtenção de resultados que correspondem às suas respectivas escolhas. Validade refere-se à importância dos dados recolhidos para a pesquisa. Operatividade corresponde a um vocabulário acessível. De acordo com Lakatos e Marconi, os questionários devem ser testados antes de sua utilização. Esse teste consiste na aplicação do questionário em uma população pequena, com o objetivo de verificar os seguintes itens: fidedignidade, validade, operatividade, ordem das questões e redação. Os questionários foram aplicados em um grupo de três pessoas, não envolvidas com a pesquisa, com o objetivo de avaliar os itens mencionados anteriormente. O resultado desse teste foi classificado como satisfatório, ou seja, não houve reclamações dos participantes acerca dos seguintes itens: objetivo da pesquisa, vocabulário, ordem e clareza das questões.

As perguntas aplicadas no questionário foram do tipo fechadas, i.e. limitadas a um grupo de escolhas fixa ou escala de avaliação. Segundo Lakatos e Marconi, este tipo de pergunta facilita o processo de tabulação e a análise de dados. Neste trabalho, foi utilizada Escala de Avaliação nas questões relacionadas aos impactos provenientes da adoção de XBRL nos processos de desenvolvimento de software e nos fatores de qualidade. Escala de Avaliação é um instrumento científico para mensurar fenômenos ou opiniões. A mesma utiliza uma escala de intensidade, a qual deverá ser avaliada pelos pesquisados, com o intuito de mostrar a sua opinião sobre um item a ser mensurado. Além disso, Escalas de Avaliação facilitam a conversão de dados qualitativos para dados quantitativos, o qual permite a realização de avaliações estatísticas [Marconi and Lakatos 2003]. A escala utilizada na pesquisa foi a Escala de Lickert. Essa escala utiliza presunções representadas por números, os quais serão utilizados na associação de opiniões. Essas numerações podem ser de cinco ou sete números: 1, 2, 3, 4 e 5 ou 1, 2, 3, 4, 5, 6 e 7. A esses números, serão adicionadas proposições, por exemplo, o número 1 pode significar muito ruim; o número 2 , ruim; o número 3 , mais ou menos; o número 4 , bom; e o número 5, muito bom. Nesse tipo de escala, quanto maior o resultado, melhor a avaliação obtida. No exemplo acima, se o resultado obtido fosse 4, significaria bom; e se o mesmo fosse 2, significaria ruim. Na pesquisa, foram atribuídas as seguintes qualificações aos respectivos números:

-o número 1: muito negativo;

•o número 2: negativo;

• número 3: nenhum impacto;

• número 4: positivo;

• o número 5: muito positivo.

Esse padrão foi aplicado nas questões relacionadas à investigação de impactos nos processos de desenvolvimento de software e nos fatores de qualidade. 


\section{Apêndice B - Procedimentos Estatísticos para Análise de Dados}

Para a análise dos resultados, identificou-se na literatura [Boslaugh and Watters 2008; Creswell 2012; Dyba 2000; Petrillo et al. 2011; Robertson 2012] a melhor forma de representação e análise para dados provenientes de questionários que usaram a Escala de Lickert. A Escala de Lickert foi aplicada nesta pesquisa para extração de informações dos participantes.

De acordo com [Petrillo et al. 2011], o uso de funções de frequência e percentagem são as melhores opções para representar dados derivados da Escala de Lickert. As funções de frequência sugeridas para representar esses tipos de dados são: quartis e moda. $O$ quartil é a quarta parte de um universo de dados, e a moda representa os valores mais frequentes em uma distribuição. Além dessas funções, [Petrillo et al. 2011] recomendam o uso de gráfico de barras e histogramas para representar graficamente informações provenientes de dados categorizados. De acordo com [Robertson 2012], a melhor forma de representação de dados oriundos da Escala de Lickert é através do uso da função moda. Robertson sugere o uso do SPSS (Statistical Package for the Social Sciences) para analisar dados categorizados. Segundo [Creswell 2012], a melhor forma de descobrir tendência em pesquisas, utilizando a Escala de Lickert, é através da Estatística Descritiva, usando as funções moda e quartis. Essas funções são utilizadas porque os dados provenientes da Escala de Lickert são categorizados. Não se pode usar a função média ou as funções de estatística inferencial para identificar tendência em dados categorizados.

"The mode is the score that appears most frequently in a list of scores. It is used when researchers want to know the most common score in an array of scores on a variable... Researchers use the mode for reporting variables with categorical variables...If we assigned numbers to each group (athletes $=4$, singers $=3$, punkers $=2$, and other $=1$ ) and calculated a mean score, $137 / 50=2.74$, it would not mean anything because no group is assigned this number. Thus, when we have categorical information, the mode reports meaningful information but the mean does not." [Creswell 2012]

Para avaliar a consistência das informações obtidas através dos questionários, [Boslaugh and Watters 2008; Dyba 2000] utilizaram o coeficiente de Alfa Cronbach [Cronbach 1951]. Esse coeficiente analisa cada item da Escala de Lickert separadamente, através da equação abaixo:

$$
\alpha=\left(\frac{n}{n-1}\right) \frac{S D_{y}^{2}-\sum\left(S D_{i}^{2}\right)}{S D_{y}^{2}}
$$

Equação para o cálculo do coeficiente de Alfa Cronbach.

Os itens da equação acima possuem as seguintes definições: 
- $n$ : significa o número de itens na escala;

- SD: significa o desvio padrão de todos os itens da escala;

- $S D_{i}$ : significa o desvio padrão de um item individual.

O resultado do coeficiente de Alfa Cronbach varia entre 0 e 1 . Segundo [Cortina 1993], um resultado do coeficiente de Alfa Cronbach acima de 0.57 é considerado consistente e confiável.

Com base no exposto acima, as funções moda foram utilizadas para analisar a tendência dos resultados, Chi-Square para validar ou refutar a hipótese nula e o coeficiente de Alfa Cronbach para analisar a consistência das informações obtidas nos questionários aplicados nessa pesquisa. 